\title{
Caracterización anatómica radial y axial de la madera de teca (Tectona grandis L. f.) plantada en Chanchamayo - Perú
} \author{
Enrique Gonzales Mora ${ }^{1}$ \\ ${ }^{1}$ Universidad Nacional Agraria La Molina, Perú. \\ ${ }^{2}$ Universidade Federal do Paraná, Curitiba, Paraná, Brasil.
}

Carlos Edmundo Chuquicaja Segura ${ }^{1 \star}$, Marcio Pereira da Rocha², Ricardo Jorge Klitzke², Héctor

\begin{abstract}
RESUMENEl objetivo del presente estudio fue describir las características anatómicas de la madera de teca (Tectona grandis $\mathrm{L}$. f.), plantada en Chanchamayo - Perú, y analizar la variación radial y axial de las dimensiones anatómicas; para ello se tomó una muestra de seis árboles de una plantación experimental de 32 años. La madera presentó grano recto ligeramente entrecruzado, textura media, frecuencia media de siete vasos. $\mathrm{mm}^{-2}$, parénquima paratraqueal escaso y parénquima apotraqueal difuso, radios homocelulares formado por células procumbentes, valor medio de cinco radios. $\mathrm{mm}^{-1}$; fibras libriformes y septadas con valores medios de $1355 \mu \mathrm{m}$ de longitud y $3 \mu \mathrm{m}$ de espesor de pared celular. En sentido axial, desde la base hacia el ápice, la longitud de fibra no presentó diferencia significativa, mientras que el espesor de pared de fibra varió significativamente en forma inversa. El diámetro de vaso mostró diferencia significativa en forma directa, mientras que la frecuencia de vasos tuvo un comportamiento inverso, a lo largo del fuste. En sentido radial, desde la médula hacia la corteza, la longitud y el espesor de pared de fibra, así como la longitud y el diámetro de vaso variaron significativamente en forma directa, mientras que la frecuencia de vasos no presentó diferencia significativa.
\end{abstract}

Palabras-clave: anatomía de la madera; dimensiones de fibra; frecuencia de vasos; teca.

\section{Radial and axial anatomical characterization of teak wood (Tectona grandis L. f.) planted in Chanchamayo - Peru}

\begin{abstract}
The objective of the present study was to describe the anatomical characteristics of teak wood (Tectona grandis $\mathrm{L}$. f.), planted in Chanchamayo - Peru, and analyze the radial and axial variation of the anatomical dimensions; for this, a sample of six trees was taken from a 32-years-old experimental plantation. The wood presented a slightly crisscrossed straight grain, medium texture, average frequency of seven vessels. $\mathrm{mm}^{-2}$, scarce paratracheal parenchyma and diffuse apotracheal parenchyma, homocellular rays formed by procumbent cells, average value of five rays. $\mathrm{mm}^{-1}$; libriform and septate fibers with mean values of $1355 \cdot \mathrm{m}$ in length and $3 \cdot \mathrm{m}$ in cell wall thickness. In the axial direction, from the base to the apex, the fiber length did not show a significant difference, while the fiber wall thickness varied significantly in reverse. The vessel diameter showed a significant difference directly, while the vessels frequency had an inverse behavior, along the shaft. Radially, from the pith to the cortex, the length and thickness of the fiber wall, as well as the length and diameter of the vessel, varied significantly directly, while the frequency of vessels showed no significant difference.
\end{abstract}

Keywords: anatomy of wood; fiber dimensions; frequency of vessels; teak.

\section{Introducción}

La madera de teca (Tectona grandis L. f.) es una de las maderas de gran demanda en el mercado mundial, gracias a sus excelentes propiedades mecánicas, estabilidad dimensional y durabilidad natural; utilizada en la construcción de barcos, producción de muebles y de objetos 
decorativos de lujo (CAMINO, 2013). La madera de teca que se comercializa en la actualidad proviene principalmente de plantaciones en diferentes zonas del mundo, tales como India, África y algunos países de Latinoamérica.

En el Perú, en los últimos años, el sector privado ha iniciado con las plantaciones de teca con fines comerciales, a pequeña escala, entre estas empresas se puede mencionar a Reforesta Perú SAC, Amazon Trading Corporation, Reforestadora Amazónica S.A., Agro Forestal Casa Blanca y Reforestadora Bánati Bosque SAC.

La madera es un material de origen biológica compleja de extrema variabilidad; sus propiedades anatómicas, físicas y mecánicas varían significativamente entre especies y entre las diferentes partes de un mismo árbol, como en el sentido médula-corteza (GONÇALVES et al., 2007; INTERIAN et al., 2011). Desde el punto de vista tecnológico, el conocimiento de la anatomía de la madera permite hacer inferencias sobre sus propiedades físicas y mecánicas, técnicas de procesamiento y utilización (INTERIAN, et al., 2011).

El desconocimiento de las características anatómicas de la madera origina que la mayoría de las veces se recurra al uso improvisado de las mismas, lo que repercute en un bajo rendimiento durante su vida útil ya que no reúnen las especificaciones requeridas para las aplicaciones a las que se destina (PEREZ; QUINTANAR, 1994). La única forma de garantizar el mejor procesamiento y uso de una especie maderable es aplicando las técnicas de transformación acorde a las características anatómicas de esa especie en particular (LEON, 2010).

Se han realizado estudios sobre la caracterización anatómica de la madera de teca y su variación en el sentido transversal de diferentes lugares de procedencia y a diferentes edades. En dichos estudios se indican que el diámetro tangencial de los vasos y la longitud de fibras aumentan en el sentido de médula a corteza, durante los primeros años y se estabilizan posteriormente, mientras que la frecuencia de vasos disminuye de médula a corteza en los primeros años y se estabiliza posteriormente (LIMA et al., 2011; GONÇALVES et al., 2007; CONEGLIAN et al., 2016; GIL et al., 2018; CARDOSO et al., 2009). En cuanto a la variación en el sentido axial se han realizado pocos estudios, en las que se indican que las dimensiones de las fibras disminuyen en sentido de la base hacia el ápice, mientras que el diámetro tangencial y frecuencia de vasos aumentan desde la base hacia el ápice (GIL et al., 2018; CARDOSO et al., 2009).

Considerando que el conocimiento de la productividad de la teca sea relevante para la recomendación de su cultivo, es importante estudiar las dimensiones de las fibras de esta madera, como uno de los parámetros de evaluación tecnológica de la especie (LIMA et al., 2011). No existen estudios publicados sobre la variabilidad anatómica de la madera de Teca proveniente de Perú, en el sentido radial y axial.

El objetivo del presente estudio fue evaluar las características anatómicas y su variación en el sentido radial y axial de la madera de teca (Tectona grandis L. f.) de 32 años de edad, cultivada en Perú.

\section{Materiales y Métodos}

\section{Datos generales de la investigación}

Para la realización del presente estudio se empleó madera proveniente de seis árboles de teca (Tectona grandis L. f.) de 32 años de edad, de una plantación experimental de 1,86 ha, con espaciamiento de 2 x 2,5 m y sin labores de manejo, propiedad de la Universidad Nacional Agraria La Molina, localizado en el fundo "Génova", distrito de San Ramón, provincia de Chanchamayo, departamento de Junín-Perú, con las coordenadas $462485 \mathrm{~m}-\mathrm{E}$ y $8773101 \mathrm{~m}-\mathrm{N}$, como se muestra en la Figura 1. La plantación se encuentra a una 
altitud promedio de $751 \mathrm{msnm}$, en la zona se registra una temperatura media anual $18^{\circ} \mathrm{C}$ a $30^{\circ} \mathrm{C}$, con una precipitación media anual de 1500 a $2000 \mathrm{~mm}$ y con un clima tropical húmedo. En la selección de los árboles se tomó en cuenta el fuste recto, que presenten buen estado fitosanitario y que tengan un diámetro a la altura de pecho (DAP) comprendido entre 25 y $30 \mathrm{~cm}$.

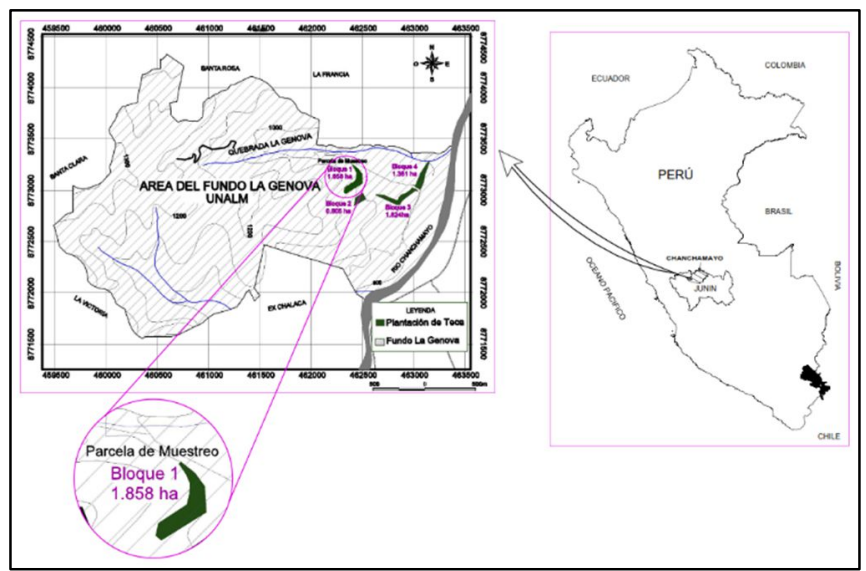

Figura 1. Localización geográfica de la plantación de Tectona grandis de 32 años

Figure 1. Geographical location of the 32-year-old Tectona grandis plantation

\section{Obtención de muestras}

De cada árbol se retiraron tres discos de madera de $5 \mathrm{~cm}$ de espesor, estableciéndose tres niveles en sentido axial, el primer nivel corresponde a la altura de corte (base), el segundo nivel corresponde a una distancia de 1,30 $\mathrm{m}$ de la base (DAP) y el tercer nivel corresponde al extremo superior de fuste (ápice), totalizando 18 discos. De cada disco se extrajo un bloque de $5 \mathrm{~cm}$ de espesor x $8 \mathrm{~cm}$ de ancho y largo variable según el radio del disco; cada bloque se dividió en tres partes iguales en dirección del radio. De la parte central de cada fracción se obtuvo un cubo de 10 x 10 x $10 \mathrm{~mm}$, debidamente orientado en sus tres planos de corte (transversal, radial y tangencial), identificados, a partir del extremo cercano a la zona de la médula, como: próximo a la médula, intermedio, y próximo a la corteza. Considerando tres niveles en sentido axial y tres niveles en sentido radial; se prepararon nueve cubos por árbol, con un total de 54 cubos, los cuales se utilizaron para la preparación de las láminas histológicas. Así mismo, al obtener cada cubo orientado de madera, también se obtuvo una fracción de madera en cada punto para el macerado, como se muestra en la Figura 2. Para la caracterización macroscópica de la madera, se extrajo de la base de cada árbol una troza de 1,3 m de largo, a partir del cual se obtuvieron tablillas de madera debidamente orientadas en corte transversal, radial y tangencial.

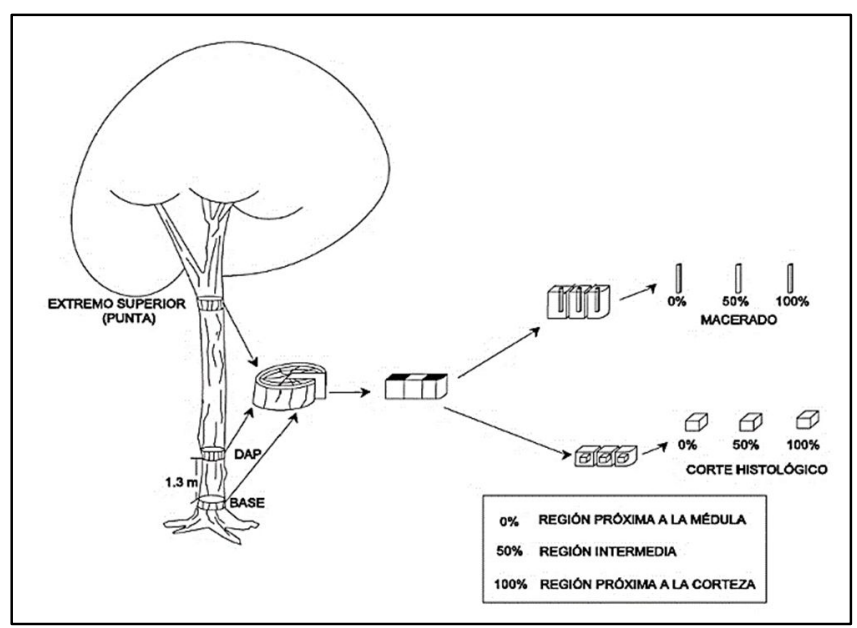

Figura 2. Esquema de muestreo de los discos en los árboles y probetas para la caracterización anatómica de la madera de teca

Figure 2. Sampling diagram of the discs in the trees and samples for the anatomical characterization of the teak wood.

\section{Estudio anatómico}

El estudio anatómico se realizó en el Laboratorio de Anatomía de la Madera de la Universidad Nacional Agraria La Molina, según la norma de procedimientos en estudios de anatomía de la madera (IBAMA, 1991). Para la identificación de las características macroscópicas, se obtuvieron tablillas de $2 \times 10 \times 15 \mathrm{~cm}$, debidamente orientadas en corte transversal, radial y tangencial, donde se evaluaron las características de color de la madera de acuerdo a Munsell, (1977), olor y sabor mediante sentido del gusto y olfato, brillo haciendo incidir una fuente de luz sobre la superficie de la madera y la textura, según el tamaño de poros, visibilidad de anillos de crecimiento, porosidad, veteado, parénquima y radios. Para 
la identificación de las características microscópicas, los cubos de madera debidamente orientados pasaron por un proceso de ablandamiento, mediante ebullición con hidróxido de sodio al tres por ciento $(\mathrm{NaOH})$, durante dos horas, luego, empleando el micrótomo de deslizamiento horizontal, se obtuvo láminas de 20-25 $\mu \mathrm{m}$ de espesor en sus tres orientaciones, transversal, radial y tangencial; las láminas fueron sometidas a un proceso de deshidratación mediante alcohol de $30^{\circ}, 60^{\circ}$ y $90^{\circ} \mathrm{GL}$ por aproximadamente 15 minutos en cada etapa. Terminado este proceso, se agregó cinco gotas de safranina y se dejó en reposo aproximadamente 20 minutos hasta conseguir un coloreado uniforme; luego se lavaron las láminas varias veces con alcohol de $96^{\circ}$ GL y se fijó el colorante con xilol. Las láminas fueron colocadas sobre el portaobjeto en el siguiente orden: transversal, radial y tangencial; antes de colocar el cubreobjetos se agregó una gota de pegamento de secado instantáneo. Considerando los seis árboles, los tres niveles en sentido axial y tres niveles en sentido radial se obtuvieron 54 láminas histológicas.

Las fracciones de madera destinadas a la obtención de fibras y vasos, pasaron por un proceso de maceración, sumergiéndolas en una solución de ácido nítrico al 33 por ciento $\left(\mathrm{HNO}_{3}\right)$, en cantidad suficiente hasta cubrirlas completamente, se las sometió a calentamiento a $80^{\circ} \mathrm{C}$ por 15 a 20 minutos, en cocina eléctrica, dentro de una campana extractora de gases. En seguida, se lavó con agua para eliminar el exceso de ácido y se agregó alcohol de $96^{\circ}$ GL y cuatro a seis gotas de safranina para coloración de las fibras y vasos y se dejó reposar por un periodo de 20 horas aproximadamente. Parte del tejido macerado se colocó en placa petri y se agregó xilol para fijar el colorante. La separación de fibras y vasos se hizo con la ayuda de una aguja hipodérmica y un microscopio. Las fibras y vasos escogidos, en cada nivel de evaluación, fueron colocados sobre una lámina portaobjeto, agregándose una gota de merkoglas, y cubriéndola inmediatamente con el cubreobjetos. Considerando las nueve posiciones de evaluación por árbol, se obtuvieron 54 láminas con fibras y vasos.

En cada posición de evaluación se hicieron 25 mediciones por cada elemento anatómico con ayuda del programa LAZ Leica Aplication, registradas en un documento excel, que incluye longitud de vaso, diámetro tangencial de vaso, número de vasos por $\mathrm{mm}^{2}$, altura de radio, ancho de radio, número de células radiales en altura y ancho de radio, número de radios por $\mathrm{mm}$ lineal, diámetro total de fibra, diámetro de lumen, longitud de fibra y espesor de pared de fibra. El espesor de pared de fibras se obtuvo a través de la Ecuación 1.

$$
E P=\frac{\mathrm{A}-\mathrm{DL}}{2}
$$

Dónde: $\mathrm{EP}=$ Espesor de pared de fibra $(\mu \mathrm{m}) ; \mathrm{A}=$ diámetro total de fibra $(\mu \mathrm{m}) ; \mathrm{DL}=$ diámetro de lumen de fibra $(\mu \mathrm{m})$.

Los elementos celulares se describieron de acuerdo a lo establecido por la International Association of Wood Anatomists (IAWA, 1989).

\section{Análisis estadístico}

La evaluación del experimento se hizo teniendo en cuenta el análisis de variancia de un diseño completo al azar con arreglo factorial $3 \times 3$ (posición radial x posición axial) para cada variable en estudio. Se verificó la respectiva distribución normal y homogeneidad de variancia. Se empleó el siguiente modelo estadístico:

$$
Y_{i j k}=\mu+\alpha_{i}+\beta_{j}+(\alpha \beta)_{i j}+\epsilon_{i j k}
$$

Donde: $\mathrm{Y}_{\mathrm{ijk}}=$ Observación correspondiente al nivel $(i)$ del factor posición radial (factor A) y al nivel $(j)$ del factor posición axial (factor B). $\mu=$ Media global. $\bullet_{i}=$ Efecto producido por el $i$-ésimo nivel del factor posición radial. $\bullet$ $=$ Efecto producido por el j-ésimo nivel del factor posición axial. $(\bullet)_{i j}=$ Efecto producido por la interacción entre el factor A y el factor B. $\bullet_{i j k}=$ Error aleatorio $i=1=$ cerca a la médula, $2=$ intermedia y $3=$ cerca a la corteza. $j=1$ = en la base, $2=$ a la altura del pecho 
(DAP) y $3=$ en el extremo superior (punta o ápice). $k=$ número de réplicas $=6 . \mathrm{H}_{0}:(\bullet)_{i j}=0$ para todo $i, j$. Es decir, los efectos de las interacciones entre los factores posición radial y posición axial, en el fuste del árbol de teca, sobre las dimensiones de los elementos anatómicos son nulos. Nivel de significancia: $\bullet=0,05$.

Se aplicó la prueba de Tukey, al nivel de 95\% de confianza entre los tratamientos y el programa estadístico MINITAB17.

\section{Resultados y Discusión}

La madera de teca (Tectona grandis L. f.) de 32 años en condición seca al aire presentó albura de color amarillo cremoso (5Y 8/2), duramen de color marrón con vetas oscuras (7,5YR 5/4), con cambio abrupto entre albura y duramen, con un espesor de albura de 2,5 cm (angosto) que representó el 22,75\% del radio; con anillos de crecimiento claramente diferenciados, limitados por bandas oscuras de forma irregular. Por cada $2,5 \mathrm{~cm}$ de radio presentó 6,25 anillos de crecimiento, con un rango de dos hasta nueve anillos siendo el menor valor cerca a la médula y el mayor valor cerca a la corteza. La madera presentó decoloración, probablemente, por oxidación debido a la exposición al aire y no presentó olor característico, presentó grano recto a ligeramente entrecruzado, de textura media, con brillo medio, con veteado de arcos superpuestos en corte tangencial causado por los anillos de crecimiento y veteado de líneas paralelas en corte radial. En cuanto a la resistencia de la madera al corte con cuchilla en sentido transversal se comportó como blanda.

Las características obtenidas con la madera estudiada son similares a las de madera de teca cultivada en otros países y de edades diferentes, señalado por algunos autores. Rodríguez-Anda et al. (2018), para madera de teca de 9, 15 y 21 años del sur de México, mencionan características anatómicas similares a lo descrito, con excepción del olor característico de la madera, lo cual podría estar relacionado con el contenido de humedad de la madera en el momento de la evaluación. Miranda et al. (2011), para madera de teca de 50 a 70 años de edad en East Timor, además de las características descritas, señalan que, el duramen es de color marrón dorado y a menudo veteado de gris o negro, con albura de color blanco amarillento, el duramen representa el 91\% del radio del tronco a la altura de 1,70 m. Así mismo indican que, el ancho promedio de albura es dos $\mathrm{cm}$ y contiene nueve anillos de crecimiento. Algunas de estas características son diferentes a las observadas en el presente estudio, lo cual podría estar relacionado con la edad de la plantación y procedencia, principalmente.

Tabla 1. Dimensión promedio de los elementos anatómicos de la madera de teca de 32 años

Table 1. Average dimension of the anatomical elements of 32-year-old teak wood.

\begin{tabular}{|c|c|c|c|c|c|c|}
\hline $\begin{array}{c}\text { Característica } \\
\text { Anatómica }\end{array}$ & Unidad & $\begin{array}{c}\text { Valor } \\
\text { promedio }\end{array}$ & $\begin{array}{c}\text { Desviación } \\
\text { estándar }\end{array}$ & $\begin{array}{c}\text { Coeficiente } \\
\text { variación }\end{array}$ & $\begin{array}{c}\text { Valor } \\
\text { mínimo }\end{array}$ & $\begin{array}{l}\text { Valor } \\
\text { máximo }\end{array}$ \\
\hline$\varnothing$ de fibra & $\mu \mathrm{m}$ & 26 & 2 & 7 & 21 & 31 \\
\hline$\varnothing$ de lumen & $\mu \mathrm{m}$ & 20 & 2 & 9 & 15 & 25 \\
\hline Espesor de Pared de fibra & $\mu \mathrm{m}$ & 3,0 & 0,3 & 11 & 2,4 & 3,8 \\
\hline Long. de fibra & $\mu \mathrm{m}$ & 1355 & 115 & 8 & 1093 & 1568 \\
\hline Long. de vaso & $\mu \mathrm{m}$ & 251 & 23 & 9 & 198 & 299 \\
\hline$\varnothing$ tang. de vaso & $\mu \mathrm{m}$ & 142 & 16 & 11 & 115 & 182 \\
\hline Frec. de vasos & $\mathrm{No}^{\circ} \cdot \mathrm{mm}^{-2}$ & 7 & 1 & 18 & 6 & 11 \\
\hline Altura de radio & $\mu \mathrm{m}$ & 684 & 93 & 14 & 467 & 881 \\
\hline Ancho de radio & $\mu \mathrm{m}$ & 58 & 10 & 17 & 40 & 84 \\
\hline Células radiales en ancho & $\mathrm{N}^{\mathrm{o}}$ & 3 & 0,4 & 13 & 2 & 5 \\
\hline Células radiales en altura & No & 28 & 4 & 14 & 18 & 34 \\
\hline Frec. de radios & $\mathrm{N}^{\circ} \cdot \mathrm{mm}^{-1}$ & 5 & 0,7 & 15 & 3 & 6 \\
\hline
\end{tabular}

$\varnothing=$ diámetro; Long. $=$ longitud; Frec. $=$ frecuencia; tang $=$ tangencial. 
En la Tabla 1 se presentan los valores medios de los elementos anatómicos obtenidos en este estudio, como parte de la descripción microscópica. La madera de teca de 32 años de edad presentó porosidad semicircular con arreglo radial que no cambia entre anillos de crecimiento, los poros se presentaron como solitarios y escasos múltiples radiales de dos hasta cuatro células (Figura 3-a), de forma redondo con diámetro tangencial promedio de $142 \mu \mathrm{m}$ en un rango de 115 a $182 \mu \mathrm{m}$, con una frecuencia media de 7 vasos. $\mathrm{mm}^{-2}$ en un rango de 6 a 11 vasos. $\mathrm{mm}^{-2}$, presentó en su interior una sustancia blanquecina. Con un elemento vascular del vaso conductor de longitud pequeño con un promedio de $251 \mu \mathrm{m}$ en un rango de 198 a $299 \mu \mathrm{m}$, con platinas de perforación horizontal con perforación simple, no presentó tílides ni engrosamiento en espiral, con escasa presencia de sustancias orgánicas (gomas). El punteado intervascular se caracterizó por ser alterno con punteaduras de forma ovaladas y dispuestas en hileras diagonales, el tamaño promedio de las punteaduras intervasculares fue de $6 \mu \mathrm{m}$, con un coeficiente de variación de 11\%. El contorno de las aperturas está incluido en el contorno de las areolas de las punteaduras (incluidas) con apertura de forma redonda o circular.

En el punteado radiovascular se observó que son iguales a las intervasculares, en forma y tamaño (Figura 3-b).

El elemento vascular del vaso conductor de la madera de teca de 32 años tiene en promedio una longitud pequeña, un diámetro tangencial mediano y una frecuencia poco numerosa, que le otorgan una textura media a esta madera; características similares a las descritas por otros autores para madera de teca de distintas edades y procedencias. Lima et al. (2011), al estudiar la anatomía de la madera de teca de 31 años en el bosque estatal de Pederneiras (São Paulo) encontraron valores promedio de diámetro tangencial de 161,28 $\mu \mathrm{m}$, longitud de $344 \mu \mathrm{m}$ y frecuencia de 8,28 vasos. $\mathrm{mm}^{-2}$. Estos valores son mayores a los resultados encontrados en el presente estudio, sin embargo, se mantienen las características de diámetro tangencial mediano, longitud pequeña y frecuencia de vasos poco numerosa. Teniendo en cuenta que las edades son similares, las diferencias podrían deberse al tipo de manejo de la plantación, la genética de las plantas y a las condiciones del sitio de producción. Moya et al. (2009), en madera de teca de 13 años de Costa Rica; Rodríguez-Anda et al. (2018), en madera de teca con edades de 9 años en el estado de Campeche, 15años en los estados de Tabasco y Campeche y 21 años en el estado de Chiapas, observaron características similares, es decir un diámetro tangencial de vaso mediano y una frecuencia de vasos poco numerosa. Moya et al. (2009), indican que el diámetro de vaso aumentó con la edad del árbol, siendo el vaso el elemento celular más influenciado por la calidad del sitio donde crecen los árboles. Rodríguez-Anda et al. (2018), indican que la procedencia influye significativamente en el aumento del diámetro tangencial de vaso.

En este estudio la madera de teca presentó parénquima visible con lupa 10X, en bandas de tipo marginal ubicado en el límite de los anillos de crecimiento; con la ayuda del microscopio se observó parénquima paratraqueal escaso y unilateral, así como parénquima apotraqueal difuso con células aisladas y escasas. Parénquima no estratificado en corte longitudinal. También presentó radios visibles con lupa 10X, homocelulares formado sólo por células procumbentes (Figura 3-c), con ancho promedio de $58 \mu \mathrm{m}$ considerado como fino, con altura promedio de $684 \mu \mathrm{m}$ y con frecuencia media de 5 radios. $\mathrm{mm}^{-1}$. El tamaño de los radios fue uniforme, con dos a cinco células de ancho y 18 a 34 células de altura (Tabla 1), en cuanto a la relación entre los radios se observó la presencia de agregados y de estructura no estratificada (Figura 3-d). 

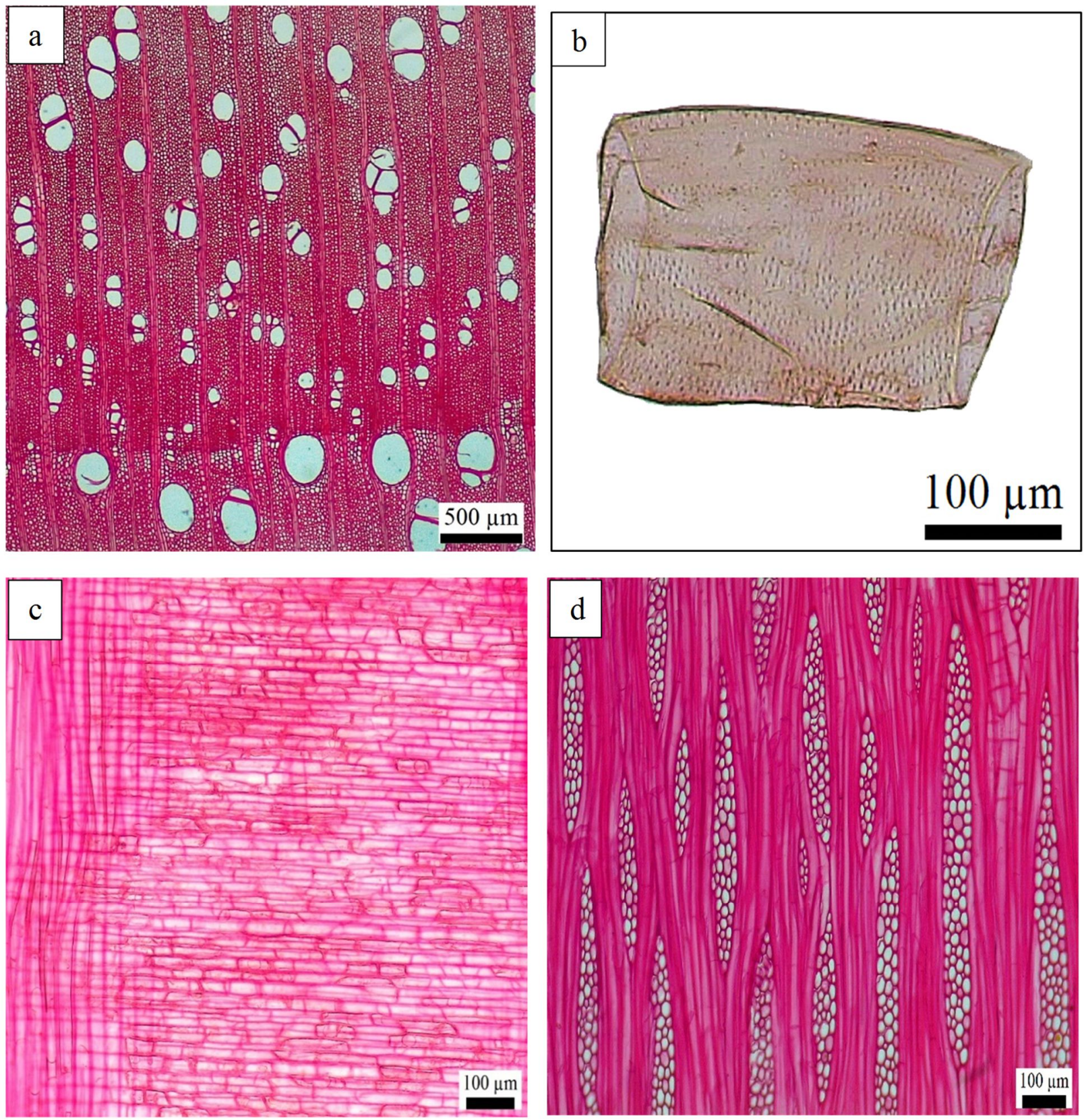

Figura 3. Estructura anatómica de la madera de teca de 32 años. a) Corte transversal mostrando la porosidad semicircular, b) Longitud y punteaduras de vaso, c) Corte radial mostrando las células procumbentes, d) corte tangencial mostrando el ancho y altura de radios.

Figure 3. Anatomical structure of 32-year-old teak wood. a) Cross section showing the semicircular porosity, b) Length and pit of the vessel, c) Radial cut showing the procumbent cells, d) Tangential cut showing the width and height of rays.

Chagas et al. (2014) señalan que, la madera de teca tiene parénquima axial paratraqueal escaso, radios multiseriados con cinco o más células en el ancho, homogéneo formado exclusivamente por células procumbentes. Por su parte Rodríguez-Anda et al. (2018) indican que la madera temprana tiene parénquima axial paratraqueal vasicéntrico escaso, radios multiseriados con dos a cinco células de ancho, radios homocelulares compuesto sólo por células procumbentes. En los estudios antes señalados, se observó el parénquima paratraqueal, así como el radio homocelular 
formado por células procumbentes, similares a lo encontrado en el presente estudio, mientras que la relación del parénquima longitudinal y el vaso difieren en los tres casos, en los que se observó parénquima paratraqueal vasicéntrico escaso, parénquima paratraqueal escaso unilateral y parénquima apotraqueal difuso con células aisladas y escasas, lo cual podría estar relacionada a la genética de las plantas y a los factores medioambientales de cada sitio.

La madera de teca del presente estudio presentó fibras con dimensiones promedio de $1355 \mu \mathrm{m}$ de longitud, $26 \mu \mathrm{m}$ de diámetro total, $20 \mu \mathrm{m}$ de diámetro de lumen y $3 \mu \mathrm{m}$ de espesor de pared de fibra, con coeficientes de variación de $8 \%, 7 \%, 9 \%$ y $11 \%$ respectivamente (Tabla 1 ). Fibras de forma libriforme, con disposición no estratificada, con punteado no visible y septadas, en promedio con dos septas por fibra (Figura 3-d). Con estas dimensiones las fibras se caracterizan como cortas y de paredes muy delgadas (IAWA, 1989).

En cuanto a las dimensiones de fibras de madera de teca, Lima et al. (2011); Thulasidas; Bhat (2012); Cardoso et al. (2009), para árboles de diferentes edades y procedencias encontraron que las fibras tienen la longitud corta (900 a $1600 \mu \mathrm{m})$ y el espesor de pared delgada a gruesa (lumen < 3(el doble del espesor de pared)) (IAWA, 1989). La longitud de fibra encontrada en el presente estudio es similar a lo reportado por los autores mencionados, mientras que el espesor de pared es menor a lo reportado por dichos autores. La diferencia del espesor de pared de fibra podría ser explicado por el bajo distanciamiento $(2 \times 2,5 \mathrm{~m})$ y por la falta de manejo de la plantación del presente estudio, así como por las condiciones de sitio. Al respecto Lima et al. (2011) señalan que el espesor de pared de las fibras en distanciamiento de 3 $\mathrm{x} \quad 1,5 \mathrm{~m}$ es significativamente menor que las de espaciamientos de 3 x 2 m y 3 x 2,5 m, así mismo Roque; Tomazello Filho (2009), para árboles de Gmelina arborea de ocho a doce años de edad encontraron que las dimensiones celulares de los elementos anatómicos son influenciados por las condiciones de manejo.

\section{Variación axial y radial}

Los resultados del análisis de variancia de las dimensiones anatómicas evaluadas de la madera de teca (Tectona grandis L. f.) de 32 años se presenta en la Tabla 2. En la posición axial se observó diferencias significativas para espesor de pared de fibra, diámetro tangencial de vaso y frecuencia de vasos, y no se observó diferencia significativa para longitud de fibra y longitud de vaso. En la posición radial se encontró diferencias significativas para longitud de fibra, espesor de pared celular, diámetro tangencial de vaso y longitud de vaso, y no se observó diferencia significativa para la frecuencia de vasos.

Tabla 2. Resumen del análisis de variancia de un diseño completo al zar con arreglo factorial para longitud de fibra (LF), espesor de pared celular (EPC), diámetro de vaso (DV), longitud de vaso (LV) y frecuencia de vasos (FV) de la madera de teca de 32 años.

Table 2. Summary of the analysis of variance of a randomized complete design with factorial arrangement for fiber length (LF), cell Wall thickness (EPC), vessel diameter (DV), vessel length (LV) and vessel frequency (FV) of 32-years-old teak wood.

\begin{tabular}{ccccccc}
\hline \multirow{2}{*}{ Fuente de Variación } & \multicolumn{5}{c}{ Cuadrado Medio } \\
\cline { 2 - 7 } & GL & LF $(\mu \mathrm{m})$ & EPC $(\mu \mathrm{m})$ & DV $(\mu \mathrm{m})$ & LV $(\mu \mathrm{m})$ & $\mathrm{FV}\left(\mathrm{n}^{\circ} \mathrm{mm}^{-2}\right)$ \\
\hline Posición Axial & 2 & $19521^{\text {n.s }}$ & $0,5973^{*}$ & $1115^{*}$ & $1227^{\text {n.s }}$ & $8,40^{*}$ \\
Posición Radial & 2 & $166797^{*}$ & $0,6885^{*}$ & $1191^{*}$ & $2260^{*}$ & $1,50^{\text {n.s }}$ \\
A x R & 4 & $5197^{\text {n.s }}$ & $0,0564^{\text {n.s }}$ & $131^{\text {n.s }}$ & $274^{\text {n.s }}$ & $1,01^{\text {n.s }}$ \\
Residuo & 45 & 6746 & 0,0667 & 182 & 417 & 1,59 \\
\hline
\end{tabular}

GL = grados de libertad; AxR = interacción de la posición axial y la posición radial 
Así mismo se encontró que no hay suficiente evidencia estadística para afirmar que el efecto de interacción de las posiciones axial y radial sobre las dimensiones anatómicas evaluadas sea diferente de cero, lo cual indica que no existe dependencia entre los factores evaluados, por tanto se acepta la hipótesis planteada con $95 \%$ de confianza, es decir, los efectos de las interacciones entre la posición radial y la posición axial en el fuste del árbol de teca, sobre las dimensiones de los elementos anatómicos, son nulos.

En la posición axial, desde la base hasta el extremo superior del fuste (ápice), el valor medio del espesor de pared de fibra en la base fue significativamente mayor a lo observado en el DAP, con una disminución de 8\%; mientras que, no se encontró suficiente evidencia estadística para afirmar que el espesor de pared en el DAP sea diferente al del ápice, sin embargo se observó una disminución de 4\%, notándose una relación inversa desde la base hacia el ápice (Figura 4-a). Este comportamiento del espesor de pared contribuye a mantener estable la resistencia mecánica de la madera a lo largo del fuste (PANSHIN; DEZEEUW, 1980). La tendencia observada para esta característica es similar a lo observado por Gil et al. (2018), quienes indican que esta disminución puede ser explicada por la necesidad de sustentación de todo el árbol. Así mismo Cardoso et al. (2009) también observaron una tendencia decreciente, precisando que dicha tendencia no siempre es lineal.

Para la longitud de fibra, no se encontró suficiente evidencia estadística para afirmar que, en la base, en el DAP y en el ápice sean diferentes; sin embargo, se observó una disminución desde la base hasta el DAP en 0,5\% y desde el DAP hasta el ápice en 4\% (Figura 4-b).

Al respecto, Gil et al. (2018) y Cardoso et al. (2009) indican que la longitud de fibra disminuye desde la base hacia el ápice, mientras que en el presente estudio se encontró un comportamiento de disminución sin llegar a ser significativo, este comportamiento de las dimensiones de fibra contribuye a la estabilidad de la calidad de la madera de teca en sentido axial.

El diámetro tangencial de vaso a la altura del DAP fue significativamente mayor a lo observado en la base, con un aumento de $10 \%$, mientras que no hubo suficiente evidencia estadística para afirmar que el del DAP sea diferente al del ápice. Sin embargo, se observó un ligero aumento desde el DAP hasta el ápice en $1 \%$, notándose un comportamiento creciente desde la base hacia el ápice (Figura 4-c).

Gil et al. (2018), indican que una de las principales células de la madera de angiospermas dicotiledóneas son los vasos, responsables del transporte de savia y penetración de preservantes y caracterizan su permeabilidad. Así mismo, para madera de teca de 7 años, señalan que el diámetro tangencial del vaso se incrementó desde la base hacia el ápice, dicho comportamiento es similar a lo encontrado en el presente estudio, a pesar de la diferencia de edad.

En relación a la longitud del vaso, se encontró que el valor medio en la base fue significativamente menor a lo observado en el DAP, con un incremento de $7 \%$ y no se observó diferencia significativa entre la longitud de vaso en el DAP. En el ápice, sin embargo, disminuyó en 3\%, de tal manera que la longitud de vaso en la base no difirió significativamente de la que se encuentra en el ápice (Figura 4-d).

El valor promedio de la frecuencia de vasos en la base fue significativamente mayor a lo observado en el DAP, con un decremento de $17 \%$ y no se observó diferencias significativas entre la del DAP y la del ápice; sin embargo se observó un incremento de $8 \%$ desde el DAP hasta el ápice, de tal manera que la frecuencia de vasos en la base y en el ápice no fueron estadísticamente diferentes; la frecuencia de vasos mostró un comportamiento inverso al de la longitud de vaso, en sentido axial (Figura 4-e). 

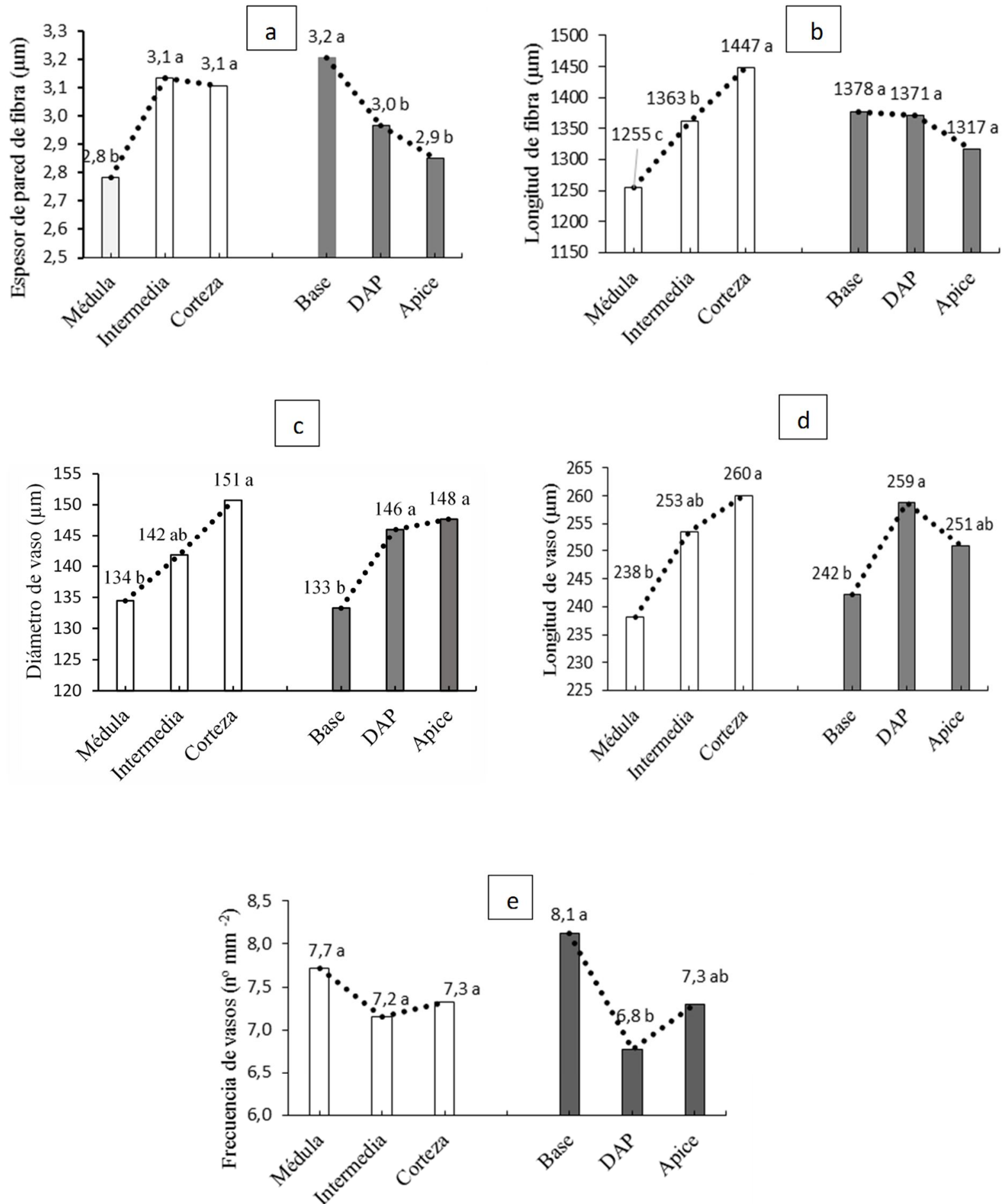

Observación: valores seguido de la misma letra no difieren entre sí según prueba de Tukey al $95 \%$ de confianza.

Figura 4. Variación radial y axial de las dimensiones de fibras y vasos de madera de teca de 32 años. a) Longitud de fibra, a) Espesor de pared de fibra, b) Longitud de fibra c) Diámetro de vaso, d) Longitud de vaso, e) Frecuencia de vasos.

Figure 4. Radial and axial variation of 32-year-old teak wood fiber and vessel dimensions. a) Fiber wall thickness, b) Fiber length, c) Vessel diameter length, d), e) Vessel frequency. 
El comportamiento de esta característica anatómica encontrado en el presente estudio, difiere de lo reportado por Gil et al. (2018) y Cardoso et al. (2009), quienes, para madera de teca, señalan que la frecuencia de vasos se incrementa de la base hacia el ápice. La diferencia en el comportamiento de esta característica anatómica podría estar relacionado con el material genético de las plantas y a factores ambientales de su entorno, tales como latitud, altitud y clima (MONTAÑO et al., 2011). Por otro lado, Giménez; López (2002), para quebracho colorado de bosques naturales en Argentina, encontraron que la frecuencia de vasos disminuye de la base hacia el ápice, mientras que el diámetro de vaso no cambia con la altura. No obstante, de ser otra especie, el comportamiento de la frecuencia de vasos guarda relación con lo encontrado en el presente estudio.

En la posición radial, el valor medio del espesor de pared de fibra en la zona intermedia fue significativamente mayor al de la zona de médula, con un aumento de $13 \%$, mientras que no se encontró diferencia significativa entre el espesor de pared de fibra de la zona intermedia con el de la zona de corteza, sin embargo, se observó una ligera disminución de 1\% (Figura 4-a). Este comportamiento, del espesor de pared, estaría indicando la presencia de madera adulta a partir de la zona intermedia hacia la corteza (BHAT et al., 2001)

La longitud de fibra en la zona de corteza fue significativamente mayor a la de la zona intermedia y ésta, a su vez, significativamente mayor a la de la zona de médula, observándose un incremento de $9 \%$, desde la médula hasta la zona intermedia, y de $6 \%$ desde la zona intermedia a la zona de corteza (Figura 4-b), comportamiento que guarda relación con la transición de madera juvenil a madera adulta (CONEGLIAN et al., 2016)

Con respecto a la variación radial de las dimensiones de fibra, de la madera de teca, se han realizado estudios para diferentes edades y procedencias, cuyos resultados corroboran lo encontrado en el presente estudio. Lima et al. (2011), Moya et al. (2009), Gil et al. (2018) y Cardoso et al. (2009) señalan que el espesor de pared y la longitud de fibra aumentan desde la médula hacia la corteza. Sin embargo, Gonçalves et al. (2007), indican que la longitud de fibra aumenta desde la médula hasta la zona intermedia y a partir de este punto, muestra cierta disminución y posterior estabilización hasta la zona de corteza. De otro lado, Portal et al. (2019), al estudiar la variación anatómica del leño de Amburana cearensis (Allemao) A.C.Sm proveniente de bosque natural en Madre de Dios, Perú, encontraron que el espesor de pared y la longitud de fibra también aumentan desde la médula hacia la corteza. Gatto et al. (2008), al estudiar la separación de madera juvenil de madera adulta de la especie Luehea divaricata de bosque natural del estado de Rio Grande do Sul, Brasil, señalan que la longitud de fibra aumenta linealmente en los primeros años, seguido de una estabilización y una ligera disminución con el aumento de la edad. Al respecto, Bhat et al. (2001) indican que las propiedades anatómicas son las que mejor predicen el límite entre madera juvenil y adulta. Considerando la edad de la plantación en el presente estudio, el comportamiento del espesor de pared de fibra en la posición radial podría estar indicando que, a partir de la zona intermedia hacia la corteza se tiene madera madura.

El diámetro tangencial de vaso en la zona de corteza fue significativamente mayor al de la zona de médula y no se encontró suficiente evidencia estadística para afirmar que el diámetro de vaso en la zona intermedia sea diferente al de la zona de médula y al de la zona de corteza, notándose un aumento gradual desde la médula hacia la corteza, en el orden de $5 \%$ desde la médula hasta la zona intermedia y $6 \%$ desde la zona intermedia hasta la zona de corteza (Figura 4-c). Al respecto, Lima et al. (2011), Gil et al. (2018) y Bhat et al. (2001), estudiando la variación radial del diámetro de vaso de 
la madera de teca, obtuvieron resultados que corroboran lo encontrado en el presente estudio. Así mismo Silva et al. (2007) y Portal et al. (2019) al estudiar la variación radial del diámetro de vaso de las especies Eucalyptus grandis, y Amburana cearensis, respectivamente, encontraron un comportamiento creciente desde la médula hacia la corteza, que es similar a lo obtenido en el presente estudio y al de otros estudios para madera de teca.

La longitud de vaso en la zona de corteza fue significativamente mayor al de la zona de médula y no se encontró suficiente evidencia estadística para afirmar que, la longitud de vaso en la zona intermedia sea diferente a la de zona de médula y a la de zona de corteza. Sin embargo, se observó un comportamiento creciente desde la zona de médula hacia la corteza, con un incremento de $6 \%$ desde la médula hasta la zona intermedia y $3 \%$ desde la zona intermedia hasta la zona de corteza (Figura 4-d), igual comportamiento al del diámetro tangencial de vaso.

Lima et al. (2011) encontraron que la longitud de vaso de la madera de teca no es influenciada por la posición radial, que es un comportamiento diferente a lo observado en el presente estudio; lo cual podría estar relacionado a la carga genética de las plantas y a factores ambientales, principalmente la altitud (MONTAÑO et al., 2011).

Para la frecuencia de vasos, no se encontró suficiente evidencia estadística para afirmar que la frecuencia de vasos en las zonas de médula, intermedia y de corteza sean diferentes, es decir, estadísticamente la frecuencia de vasos no varió en la posición radial. Sin embargo, se observó una ligera disminución desde la médula hasta la corteza, en el orden de 5\% (Figura 4-e).

Lima et al. (2011), Gil et al. (2018), Moya et al. (2009) y Cardoso et al. (2009), al estudiar la variación radial de la frecuencia de vasos de la madera de teca, mencionan un comportamiento decreciente en el sentido médula a corteza, comportamiento que es similar a lo encontrado en el presente estudio. Por otro lado, León; Quintero (2007) y Sette et al. (2009), al estudiar la variación radial de la frecuencia de vasos de las especies Gmelina arborea Roxb y Eucalyptus grandis, respectivamente, también mencionan que la frecuencia de vasos disminuye en el sentido médula a corteza.

De acuerdo a las características anatómicas observadas y a su respectivo comportamiento en sentido axial y radial, se puede señalar que las características anatómicas de la madera de teca producida en Perú son similares a las de madera de teca producida en otros países, en algunos casos a diferentes edades; sin embargo, se debe evaluar las propiedades mecánicas de esta madera para definir su mejor uso alternativo.

\section{Conclusiones}

La madera de teca (Tectona grandis L. f.), proveniente de una plantación de 32 años de la zona de Chanchamayo, Perú, tiene características anatómicas similares a la madera de teca producidas en otros países.

No hay interacción entre las posiciones axial y radial sobre las dimensiones de los elementos anatómicos de la madera de teca.

En sentido axial, el espesor de pared de fibra y la frecuencia de vasos disminuyen y la longitud y diámetro tangencial de vaso se incrementan, mientras que la longitud de fibra no es influenciada por la posición axial.

En el sentido radial, el espesor de pared de fibra, la longitud de fibra, el diámetro de vaso y la longitud de vaso se incrementan, mientras que la frecuencia de vasos no es influenciada por la posición radial. 


\section{Referencias}

BHAT, K. M.; PRIYA, P. B.; RUGMINI, P. Characterization of juvenile wood in teak. Wood Science and Technology, Springer-Verlag, v. 34 n. 6, p. 517-532, 2001.

CAMINO, R. de. Las plantaciones de teca en América Latina: mitos y realidades/Ronie de Camino. -Turrialba, C. R.: CATIE, 2013. 392p. - (Serie técnica. Informe técnico/CATIE, No. 397).

CARDOSO, S.; SOUSA, V.; QUILHÓ, T.; PEREIRA, H. Variabilidade anatômica da teca (Tectona grandis) de TimorLeste. In: CONGRESSO FLORESTAL NACIONAL: A FLORESTA NUM MUNDO GLOBALIZADO, 6, 2009, Ponta Delgada. Anais... Ponta Delgada: SCPF, 2009. p. 536543.

CHAGAS, S. F.; EVANGELISTA, W. V.; SILVA, J. C.; CARVALHO, A. M. M. L. Propriedades da madeira de teca de diferentes idades e oriundas de desbaste, Ciência da Madeira (Brazilian Journal of Wood Science), v. 5, n. 2, p. 138-150, 2014.

CONEGLIAN, A.; CAMPOS, N. P.; SETTE JUNIOR, C. R.; DA SILVA, A. R.; DE SOUSA JR, A. D.; PEREIRA, I. M. Using segmented linear regression to identify juvenile and mature wood of Tectona grandis. International Journal of Current Research, v. 8, n. 11, p. 42021-42024, 2016.

GATTO, D. A.; HALESEIN, C. R.; BUligON, E. A.; COLEGARI, L.; STANGERLIN, D. M.; OLIVEIRA L. S.; SANTINI, E. J. Estimativa da idade de segregação do lenho juvenil e adulto por meio de parámetros anatómicos para madeira de Luehea divaricata Mart, Ciência Florestal, Santa María, v. 18, n. 4, p. 335-540, 2008.

GIL, J. L. R. A.; BARBOZA, F. S.; CONEGLIAN, A.; DA SILVA, M. F.; DE MORAES, M. D. A.; SETTE JUNIOR, C. R. Características físicas e anatómicas da madeira de Tectona grandis L. f. aos 7 anos de idade. Revista de Ciências Agrarias, v. 41, n. 2, p. 529-538, 2018.

GIMÉNEZ, A. M.; LÓPEZ, C. R. Variación longitudinal de los elementos del leño en Schipnosis quebracho-colorado (Schlecht.) Barkl. et Meyer Anacardiaceae. Madera y Bosques. v. 8, n. 2, p. 27-38, 2002.

GONÇALVES, M. P.; COFFlER, R.; CARVAlHO, A. M.; GARCIA, R. A. Variação radial da densidade básica e comprimento das fibras da madeira da Tectona grandis $\mathrm{L}$. Floresta e Ambiente, v. 14, n. 1, p. 70-75, 2007.
IAWA COMMITTEE. IAWA list of microscopic features for hardwood identification. IAWA Bulletin, n.s., v.10, n.3, p. $219-332,1989$.

IBAMA - Instituto Brasileiro do Meio Ambiente e dos Recursos Naturais Renováveis. Normas de procedimentos em estúdios de anatomía da madeira: I. Angiospermae, II. Gimnospermae. Brasil. 19 p. 1991.

INTERIÁN-KU, V. M.; BORJA DE LA ROSA, M. A.; VALDEZ-HERNÁNDEZ， J. I.; GARCIA-MOYA， E.; ROMERO-MANZANARES, A.; VAQUERA-HUERTA, H. Características anatómicas y propiedades físicas de la madera de Caesalpinia gaumeri Greenm en Dzan, Yucatán, Madera y Bosques, v. 17, n. 1, p. 23-36, 2011.

LEON-H, W. J. Anatomía y densidad o peso específico de la madera, Revista Forestal Venezolana, año XLIV, v. 54, n. 1, p. 67-76, 2010.

LEON-H, W. J.; QUINTERO-M, M. A. Variabilidad de los vasos en Gmelina arborea Roxb. (Verbenaceae) proveniente de plantaciones de estado Portuguesa (Venezuela), Revista Forestal Venezolana, v. 51, n. 1, p. 97-113, 2007.

LIMA, I. L.; GARCIA, R.; LONGUI, E. L.; FLORSHEIM, S. M. B. Dimensões anatômicas da madeira de Tectona grandis Linn. em função do espaçamento e da posição radial do tronco. Scientia Forestalis, Piracicaba, v. 39, n. 89, p. 61-68, 2011.

MIRANDA, I.; SOUSA, V.; PEREIRA, H. Wood properties of teak (Tectona grandis) from a mature unmanaged stand in East Timor. Journal of Wood Science, v. 57, n. 3, p. 171-178, 2011.

MONTAÑO, A. S.; CAMARGO, R. S.; GRETHER, R.; DE LA PAZ, P. C. La madera en función del ambiente: un análisis ecoanatómico. Elementos: Ciencia y cultura, v. 18, n. 83, p. 19-23, 2011

MOYA, R.; BERROCAL, A.; SERRANO, J. R.; TOMAZELLO FILHO, M. Variación radial de la anatomía, densidad y durabilidad de la madera de teca (Tectona grandis) procedente de dos calidades de sitio y dos regiones climáticas de Costa Rica. Forest Systems, Madrid, España, v. 18, n. 2, p. 119-131, 2009.

MUNSELL COLOR CHARTS FOR PLANT TISSUES. Munsell color. Kollmorgen Corporation Baltimore, Maryland. 17 p. 1977. 
PANSHIN, A. J.; DE ZEEUW, C. Textbook of wood technology. Ed. 4. Mc Graw Hill Book Company Inc. New York. 723 p. 1980.

PEREZ, O. C.; QUINTANAR, I. A. Características anatómicas de la madera de cinco especies del estado de Jalisco, México, Acta Botánica Mexicana, n. 27 p. 75-87, 1994.

PORTAL-CAHUANA, L. A.; DE FIGUEREIDO, J. V.; CAMARGO-PASE, J. H.; VIEIRA DOS SANTOS, G. C.; OLIVEIRA-LIMA, D.; ALVES-RAMOS, L. M.; FIGUEREIDO DO CARMO, J. Variabilidad radial física y anatómica del leño de árboles de Amburana cearensis (Allemao) A.C.Sm. Colombia Forestal, v. 22, n. 1, p. 17-26, 2019.

RODRIGUEZ-ANDA, R.; FUENTES-TALAVERA, F. J.; SILVA-GUZMAN, J. A.; PALACIOS-JUAREZ, H.; TURRADO-SAUCEDO, J. Relationship of the cellular structure of teak wood (Tectona grandis L. f.) with different ages and growth sites. Revista Chapingo Serie Ciencias Forestales y del Ambiente, v. 24, n. 1, p. 101-114, 2018.

ROQUE, R. M.; TOMAZELHO FILHO, M. Variação radial da estrutura anatómica do lenho do árvores de Gmelina arborea em diferentes condições de clima e de manejo na Costa Rica. Scientia Forestalis, Piracicaba, v. 37, n. 83, p. 273$285,2009$.

SETTE JUNIOR, C. R.; TOMAZELHO FILHO, M.; LOUSADA, J. L.; LACLAU, J. P. Efeito da aplicação fertilização nitrogenada e lodo de esgoto nas características da madeira juvenil de árvores de Eucalyptus grandis, Cerne, v. 15, n. 3, p. 303-312, 2009.

SILVA, J. C.; TOMAZELHO-FILHO, M.; OLIVEIRA, J. T. S.; CASTRO, V. R. Influência da idade e da posição radial nas dimensões das fibras e dos vasos da madeira de Eucalyptus grandis Hill ex Maiden, Revista Árvore, Viçosa-MG, v. 31, n. 6, p. 1081-1090, 2007.

THULASIDAS, P. K.; BHAT, K. M. Mechanical properties and wood structure characteristics of 35-year old homegarden teak from wet and dry localities of Kerala, India in comparison with plantation teak. Journal Indian Academy Wood Science, Springer, v. 9, n. 1, p. 23-32, 2012. 